\title{
Current status and future prospects for laboratory study of angular momentum transport relevant to astrophysical disks
}

\author{
Hantao Ji \\ Princeton Plasma Physics Laboratory, \\ Princeton University, \\ Princeton, New Jersey, U.S.A. \\ email: hji@pppl.gov
}

\begin{abstract}
A concise review of the past and ongoing laboratory experiments on rotating flows and the associated angular momentum transport relevant to astrophysical disks is given in three categories: hydrodynamic, magnetohydrodynamic, gas and plasma experiments. Future prospects for these experiments, especially for those directly relevant to the magnetorotational instability (MRI), are discussed with an emphasis on a newly proposed swirling gas and plasma experiment.
\end{abstract}

Keywords. accretion disks, hydrodynamics, magnetohydrodynamics (MHD), instabilities, plasmas, turbulence, methods: laboratory

\section{Introduction}

Accretions disks consist of gas, dust, and plasma rotating around and gradually falling onto a central object. Many important astrophysical phenomena take place in accretion disks ranging from formation of stars and planets in proto-star systems, the mass transfer and energetic activity in binary star systems, to efficient energy releases in quasars and active galactic nuclei. A common puzzle across all these kinds of accretion disks is why accretion occurs fast compared to the predictions based on classical viscosity by many orders of magnitudes. This is equivalent to the question of how angular momentum is rapidly transported radially outwards. Cause of this rapid transport is generally attributed to the presence of rigorous turbulence in the accretion disk (Shakura and Sunyaev, 1973).

The difficulty of the problem, however, originates from the fact that there exist no known robust linear hydrodynamic instabilities in Keplerian disks where angular velocity, $\Omega$, scales as radius, $R^{-3 / 2}$. The well known Rayleigh stability criterion (Rayleigh, 1916) against centrifugal instability $d\left(R^{2} \Omega\right) / d R>0$ is satisfied. Therefore, there exist only two main mechanisms to generate turbulence in accretion disks: (1) nonlinear hydrodynamic instabilities and (2) linear magnetohydrodynamic instabilities, and more specifically Magnetorotational Instability or MRI. The possibility of nonlinear hydrodynamic instabilities was first pointed out by Zeldovich (1981) and followed up by Richard and Zahn (1999) based on experiments of Wendt (1933) and Taylor (1936) in TaylorCouette flows (see Sec. 2). The idea is similar to what has been learned from pipe flows where subcritical transition was shown as the mechanism to generate turbulence despite linear stability (e.g., Eckhardt 2007). The argument is that in cool disks such as starforming disks where ionization fraction is too low for the magnetic field to have any effects on disk dynamics, these non-magnetic, nonlinear hydrodynamic instabilities may be the only available mechanisms to generate the required turbulence. However, these 
nonlinear instabilities have not been demonstrated conclusively under astrophysically relevant conditions either theoretically, numerically, or experimentally.

In hot, thus sufficiently ionized accretion disks such as in binaries, the magnetic field effects are widely expected in generating turbulence (Shakura and Sunyaev, 1973). The well-accepted scenario there is that the turbulence is generated by the MRI, originally discovered by Velikhov (1959) and Chandrasekhar (1960) and re-discovered for astrophysical applications by Balbus and Hawley (1991), and (1998). The MRI is a linear ideal MHD instability which grows locally in Keplerian disks on the orbital timescale in a weak magnetic field. Because of the robustness of the MRI in numerical simulations there is little doubt on the existence of MRI, although it has not been positively confirmed either observationally or experimentally in the laboratory. The key problem currently under hot debate, however, becomes what turbulent viscosity the MRI can generate to transport angular momentum (Lesur and Longaretti, 2007; Fromang et al. 2007). Below we list the outstanding questions for angular momentum transport in astrophysical disks:

- Can nonlinear hydrodynamic turbulence efficiently transport angular momentum?

- How does the MRI saturate to generate angular momentum transport?

- How does the transport scale with Reynolds number?

- Are Hall effects and ambipolar diffusion important for the MRI and its saturation?

- Can kinetic effects significantly modify angular momentum transport?

- How do radiation and relativity affect the MRI and its saturation?

In principle, all of these issues (except the last one) can be studied in detail in the laboratory. We note that the role of laboratory experiments here is not to directly simulate astrophysical phenomena themselves, but to study their fundamental underlying physics. Therefore, numerical simulations combined with analytic theory play a crucial role in guiding laboratory experiments, in interpreting their results, and in making actual connections to astrophysical phenomena.

In the following Sections, three categories of experiments will be discussed in terms of their current status and future prospects in addressing the above issues.

\section{Hydrodynamic Experiments}

Current Status. Study of rotating flows between two concentric cylinders goes back to Couette (1890) who used it as a way to measure viscosity of water. Taylor (1923) significantly expanded the horizon of the Couette flow by successfully applying linear theory of Navier-Stokes equations using nonslip boundary conditions to accurately explain experimentally observed stability conditions for the centrifugal instability (Rayleigh, 1916). All subsequent work of Taylor-Couette flows focused on this centrifugal instability and its subsequent bifurcations eventually to full turbulence, except two specific work by Wendt (1933) and Taylor (1936) on linearly stable flows with $d \Omega / d R>0$ which unfortunately are irrelevant to accretion disks, but are used in Richard and Zahn (1999). The relevant flows are quasi-Keplerian flows where $d \Omega / d R<0$ and $d\left(R^{2} \Omega\right) / d R>0$.

First modern hydrodynamic experiments on quasi-Keplerian were performed by Richard (2001) and Beckley (2002). Richard found signs of turbulence in quasi-Keplerian flows based on a visualization technique, but the end caps were either corotating with the outer cylinder (the "Ekman" configuration) or a half of the end caps corotating with the inner cylinder while the other half with the outer cylinder (the "Split" configuration). Both configurations are subject to Ekman circulation (Kageyama et al., 2004) which can efficiently transport angular momentum and thus modify flow profile significantly away from the so-called Couette profiles (which are solutions with ideal axial boundary conditions). Thus, the observed turbulence is subject to the interpretation of Ekman 

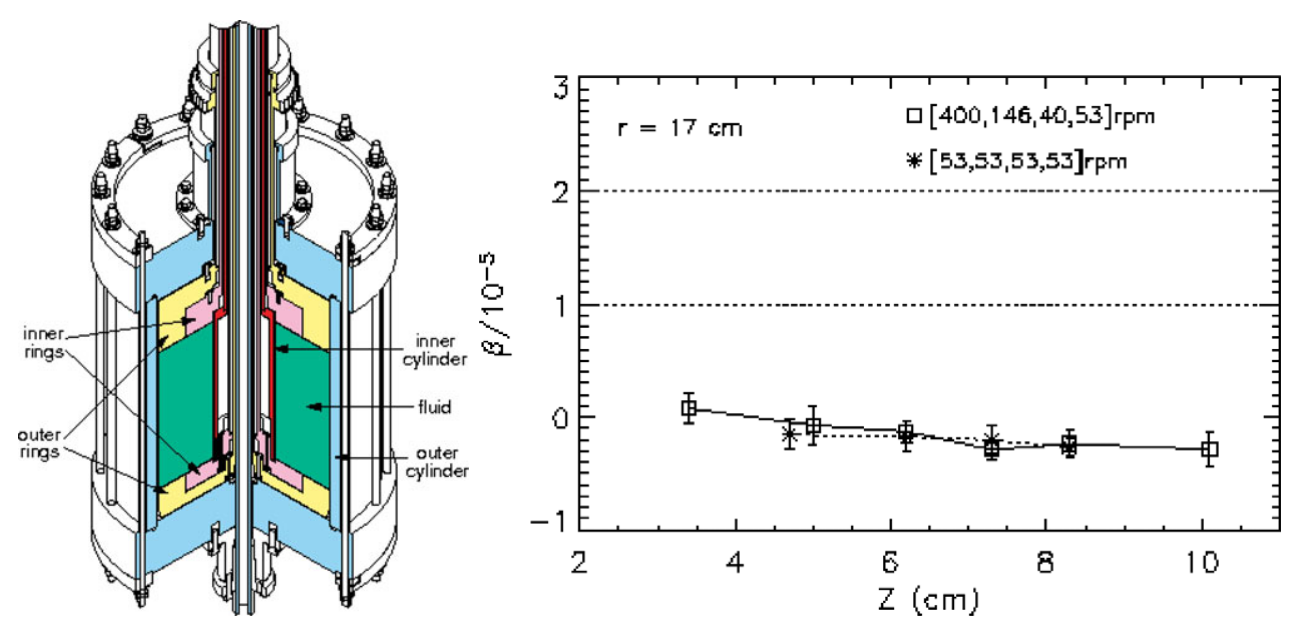

Figure 1. (left) End caps are divided into two rings in the Princeton MRI experiment; each of them are driven at the different speed to minimize the Ekman effect. (right) Measured angular momentum transport in quasi-Keplerian flows is indistinguishable from that in solid body flow with proper boundary controls, indicating no signs of turbulence.

circulation, rather conclusive evidence of nonlinear instability. Beckley (2002) measured torque in quasi-Keplerian flows in an Ekman configuration, and concluded that the measured torque was consistent with the expected angular momentum transport by Ekman circulation. Finally, higher transport levels inferred from torque measurements in quasiKeplerian flows with end caps corotating with the outer cylinder in a more recent study Paoletti and Lathrop (2010) are also subject to the interpretation based on the Ekman circulation.

To minimize Ekman circulation, a Taylor-Couette device with active controls of axial boundary conditions was built, as shown in Fig.1(left) (Burin et al., 2006; Schartman et al., 2009). Each end cap is split into two rings, and each ring is driven separately at a selectable speed, different from those of the inner and outer cylinders. By choosing appropriate speeds for these rings, Couette profiles can be accurately restored, indicating minimization of Ekman circulation. Local Reynolds stress for angular momentum transport were determined ( $\mathrm{Ji}$ et al., 2006) through correlating radial and azimuthal velocity components measured by dual Laser Doppler Velocimetry (LDV). It was found that there are no signs of turbulence in quasi-Keplerian flows with boundary controls at Reynolds numbers up to $2 \times 10^{6}$ (Ji et al., 2006; Schartman et al., 2010), as shown in Fig.1(right). The measured transport efficiency is significantly below the requirements from the observed accretion rates. The conjectured nonlinear instability either has happened but at undetectable levels or does not happen until a Reynolds number higher than $2 \times 10^{6}$ is reached. Since the higher the critical Reynolds the less effective the turbulence will be (Lesur and Longaretti, 2005), it is highly unlikely that hydrodynamic turbulence is responsible for fast accretion even for cool disks.

Future Prospects. Despite the recent progress summarized above, an outstanding question remains as to why quasi-Keplerian flows can be so stable even at very high Reynolds numbers. This question may be related to the general observations of stable atmospheric vortices, such as hurricanes and tornados. Detailed studies of nonlinear stability of quasi-Keplerian flows are planned on a newly constructed, mechanically improved Taylor-Couette flow device at Princeton. 
Another question regarding angular momentum transport in hydrodynamics concerns coherent wave activity, such as Rossby waves (Rossby et al., 1939), in the context of accretion disks (e.g. Lovelace, 1999). Such waves exist due to the presence of radial variations of Coriolis force and can grow by flow shear into a large amplitude as seen in Earth's atmosphere and oceans. It is planned on the new Princeton hydrodynamic experiment to study Rossby wave excitation using radially varying axial height as in the earlier experiments (Sommeria et al., 1988) but focusing on the associated angular momentum transport. Finally, compressible gas dynamics of quasi-Keplerian flows can be studied in the proposed Princeton plasma MRI experiment described in Sec.4.

\section{Magnetohydrodynamic (MHD) Experiments}

Current Status. Earlier MHD experiments using liquid metal in Taylor-Couette flow focused on stabilization of centrifugal instability by a magnetic field (Donnelly and Ozima, 1960). Modern MHD Taylor-Couette flow experiments were not proposed (Ji et al., 2001; Rüdiger and Zhang, 2001 until a decade after the rediscovery of the MRI. The standard setup for such an experimental realization (Ji et al., 2001; Goodman and Ji, 2002 ) is to apply a sufficiently strong axial magnetic field to destabilize an otherwise stable, quasi-Keplerian flow. To date, however, a definitely positive identification of the MRI in the standard setup has not been realized due to technical difficulties in achieving sufficiently fast speeds while maintaining mechanical integrity of the device, such as accurate controls of end rings (Schartman et al., 2009). Currently, the highest speeds achieved in the Princeton MRI experiment are right on the edge of the required speeds to destabilize the MRI.

Nonetheless, there are two noteworthy milestones on the long journey towards demonstrating MRI in the laboratory. The first one is based on an experimental setup using spherical Couette flow (Sisan et al., 2004). Before an axial magnetic field is imposed, the
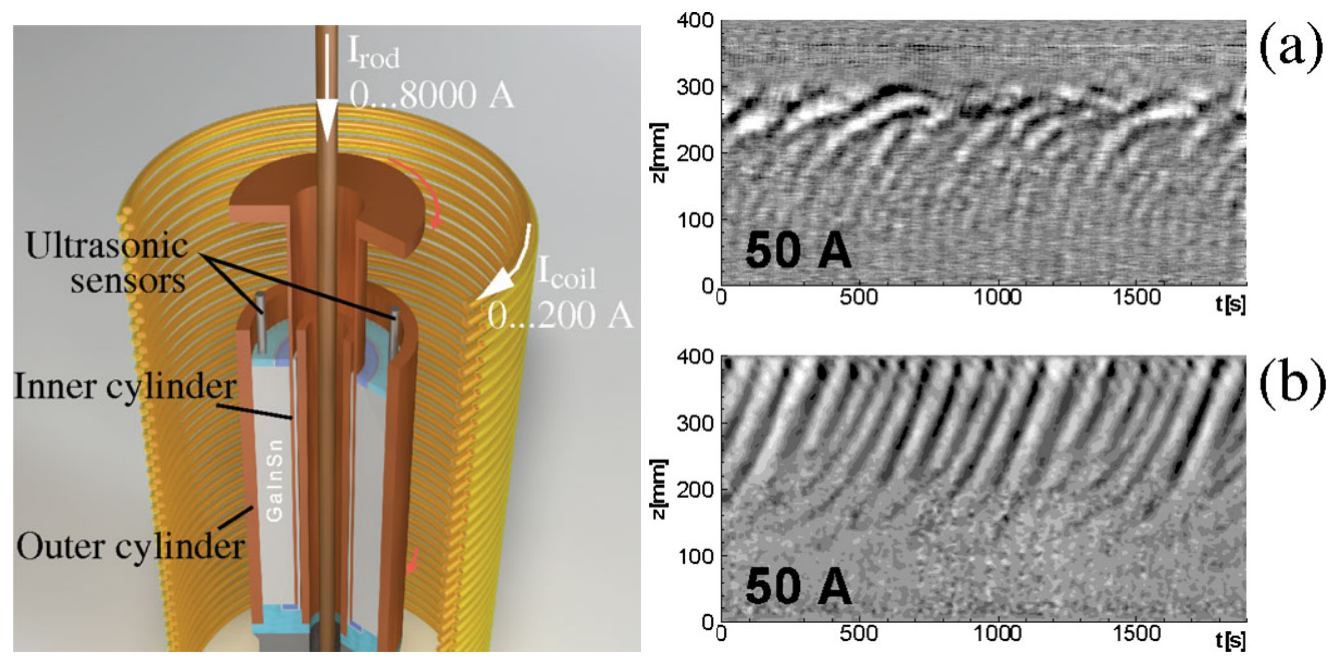

Figure 2. (left) PROMISE 2 device where end caps are split into two pieces with each attached to a cylinder, in contrast to PROMISE 1 (not shown) where the lower end cap rotates with the outer cylinder with the upper cap stationary. Both axial and azimuthal fields are imposed externally. (right) Measured axial velocity perturbations for PROMISE 1 (a) and PROMISE 2 (b) in quasi-Keplerian flows. Upward traveling waves were significantly disrupted by Ekman circulation in PROMISE 1 while much less so in 2, consistent with simulations. Figure courtesy of F. Stefani. 
flow is hydrodynamically unstable since outer sphere is stationary. The flow exhibits instabilities with finite magnetic components of different nonaxisymmetric spherical modes at certain strengths of the imposed field, accompanied by increases in torque required to rotate the inner sphere. These instabilities were interpreted as the MRI which has similar expectations. Recently, a 3D MHD numerical study (Gissinger et al., 2010) has been performed in a similar setup but necessarily at lower Reynolds numbers. Most experimental results were successfully reproduced; the resultant instabilities are identified as instabilities of a shear layer, called the Schercliff layer (Gissinger et al., 2010), induced by a combination of sphere boundaries and the applied magnetic field. The Schercliff layer instabilities, however, are probably irrelevant to astrophysical disks due to the absence of these special boundaries there. They may be more relevant to planetary cores where similar boundaries and magnetic field can coexist. Interestingly, coherent oscillations in the external magnetic field (Nornberg et al., 2010) and in the internal flow (Roach et al., 2010) were also identified in the Princeton MRI experiment when a sufficiently strong magnetic field is imposed even at relatively low magnetic Reynolds numbers. These oscillations resemble the predicted Schercliff layer instabilities, but a positive identification is in order.

Hollerbach and Rüdiger (2005) reported a variation of the MRI, called the Helical MRI (HMRI), can be destabilized at much lower Reynolds numbers by imposing an azimuthal magnetic field, in addition to the standard axial magnetic field. The HMRI is an inductionless instability since it survives at the vanishing magnetic Reynolds number limit, in contrast to the standard MRI. It exhibits a form of traveling waves (Liu et al., 2007) which have been subsequently reproduced in the PROMISE experiments (Stefani et al., 2006; Stefani et al., 2009). Once again, the importance of axial boundary conditions was demonstrated: in the original device with significant Ekman circulation the traveling waves were disrupted by returning radial flow slightly above the mid height, while the traveling waves propagate smoothly throughout the flow when Ekman circulation is reduced by modified end caps, see Fig.2. The nature of the HMRI has been identified as a weakly destabilized inertial oscillation, which is unfortunately stable for Keplerian flows (Liu et al., 2006) although the role of some special radial boundary conditions is still subject to further clarifications (Rüdiger and Hollerbach, 2007; Liu, 2008).

Future Prospects. An experimental campaign is currently underway at Princeton to search for the standard MRI at higher Reynolds numbers considerably above the predicted threshold. Probably there is little doubt that some kind of magnetic instability will be found in the new regimes. Rather, the real challenge there is whether we can distinguish the MRI from the aforementioned boundary-induced instabilities which exhibit similar behaviors. This speaks for the crucial importance of numerical simulations and theoretical analyses as exemplified by the cases of Schercliff layer instability and HMRI.

A next challenge for laboratory experiments, also for numerical simulations, is extrapolating the results to astrophysically relevant parameters which are certainly beyond their reach. Therefore, an important step is for experiments to access a wide range of parameters so that meaningful scalings can be established, understood, and thus extrapolated with confidence to astrophysically relevant regimes. To this extent, Princeton liquid metal MRI experiment is planning an upgrade to broaden the accessible magnetic Reynolds numbers but still under well controlled boundary conditions. Separately, an ambitious facility, named "DRESDYN" (Stefani, 2010), is under planning in Dresden, Germany, for an even wider range of accessible parameters. Currently, other related instabilities, the so-called azimuthal MRI under a purely azimuthal magnetic field (Hollerbach et al., 2010) and current-driven Tayler Instability (Rüdiger and Schultz, 2010), are also under investigation in Dresden. 


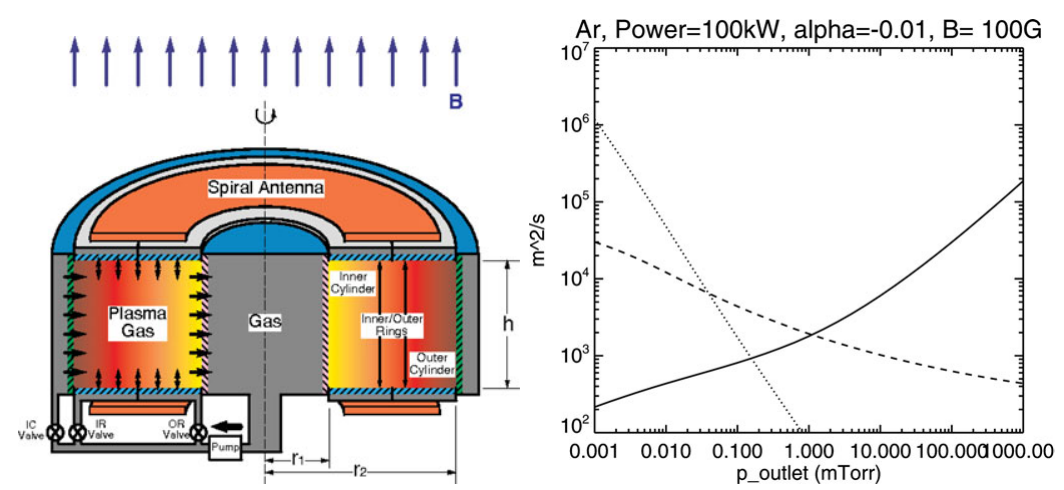

Figure 3. (left) Proposed Princeton Plasma MRI experiment where swirling gas flow is setup by an injection-pumping system in an annular geometry enclosed by two concentric cylinders and end caps. Gas is injected tangentially from the outer cylinder and is pumped out from the inner cylinder. Gas injection and pumping at the end caps are used to adjust rotation profile through adding or removing angular momentum. Spiral antennas are used to transmit RF power into the experiment to ionize the gas with a desirable degree of ionization. (right) Calculated Ohmic (solid line), Hall (dashed line), and ambipolar (dotted line) diffusivilities as a function of gas pressure at the outlet. Different regimes can be accessed by selecting appropriate pressures.

Another potentially interesting possibility is resurrection of nonlinear transition to turbulence by a weak and diffusive magnetic field to break hydrodynamic constraints in otherwise stable hydrodynamic flows at large Reynolds number. In fact, resistive HMRI is a linear counterpart of such transitions. Numerical tests of such transitions could be helpful in determining the required initial perturbations and magnetic field strengths for such transitions.

\section{Gas and Plasma Experiments}

Current Status. There exist a number of physical effects absent in incompressible hydrodynamic or liquid metal experiments but they could be important in accretion disks. A subset of such effects can potentially be studied in gas and plasma experiments: MHD physics at larger magnetic Prandtl numbers than available in liquid metals, non-baroclinic effects due to non-coplanar temperature and pressure gradients, Hall effects due to different ion and electron masses, ambipolar diffusion due to ion-neutral collisions, and kinetic effects such as anisotropic viscosity, anisotropic thermal conduction, and microinstabilities.

There existed quite a few rotating plasma experiments, but few of them were specifically targeted for MRI studies. Within the MHD framework, if a magnetic field is used to confine plasma from the first place, such as in tokamaks, the plasma $\beta$ will be necessarily less than unity, and thus stable to the MRI. Instabilities found in these experiments are mostly electrostatic in nature similar to classical interchange instabilities. In two recent rotating plasma experiments (Wang et al., 2008; Teodorescu et al., 2010), magnetic fluctuations were detected but their sources are unidentified among many possible competing candidates other than the MRI.

Future Prospects. There are two active lines of approach to study the MRI in plasmas. The approach under development at Wisconsin, called the Plasma Couette Experiment (Collins et al., 2010), uses the multipolar magnetic configuration of strong permanent magnets mounted on a cylinderical surface to confine high beta plasma inside. The plasma rotation is driven by biasing the plasma perpendicular to the local magnetic field. 
The second approach being proposed at Princeton, called the Plasma MRI experiment (Ji, 2011), is based on a prototype experiment using helicon wave plasmas (Ji et al., 2007). The idea is to set up a swirling gas flow with desired profiles by utilizing a gas injection-pumping system shown in Fig.3. Spiral antennas are used to transmit RF power into the experiment to ionize the gas with a desirable degree of ionization. A wide range of outstanding issues can be studied in such device, including: nonlinear hydrodynamic instability, baroclinic instability with axial or azimuthal temperature gradient, MRI in weakly ionized plasmas with Hall effect and ambipolar diffusion.

\section{Conclusions}

A concise review is given about three kinds of rotating flows in the laboratory: hydrodynamic, MHD, and gas/plasma experiments. Each of these can attack a unique set of outstanding questions in angular momentum transport relevant to astrophysical disks. Hydrodynamic experiments showed that nonlinear hydrodynamic turbulence is unlikely responsible for the turbulence in Keplerian disks while the mechanisms for stability of such a flow are still unclear. MHD experiments using liquid metal are on the verge of realizing the standard MRI while the related helical MRI has been already demonstrated. A challenge there is how to distinguish the MRI from boundary-induced instabilities, such as Schercliff layer instability. Gas and plasma MRI experiments are under development, and rich physics is expected be learned from these experiments such as Hall effect and ambipolar diffusion. In all of these cases, close interactions and collaborations with theory and simulation are crucial in understanding experimental results and in developing scalings applicable to astrophysical disks.

\section{References}

Balbus, S. A. \& Hawley, J. F., Astrophys. J., 376:214-222, 1991.

Balbus, S. A. \& Hawley, J. F., Rev. Mod. Phys., 70:1-53, 1998.

Beckley, H., PhD thesis, New Mexico Institute of Mining and Technology, 2002.

Burin, M. J., Schartman, E., Ji, H., R. Cutler, P. Heitzenroeder, W. Liu, L. Morris, \& S. Raftopolous. Experiments in Fluids, 40:962-966, 2006.

Chandrasekhar, S., Proc. Nat. Acad. Sci., 46:253-257, 1960.

Collins, C., Katz, N., Weisberg, W., Clark, M., Wallace, J., \& Forest, C., Bull. Am. Phys. Soc., 55:BAPS.2010.DPP.NP9.68, 2010.

Couette, M. M., Ann. Chim. Phys., 6:433-510, 1890.

Donnelly, R. J. \& Ozima, M., Phys. Rev. Lett., 4:497-498, 1960.

Eckhardt, B., Schneider, T. M., Hof, B., \& Westerweel, J., Annual Review of Fluid Mechanics, 39(1):447, 2007.

Fromang, S, Papaloizou, J., Lesur, G., \& Heinemann T., Astron. Astrophys., 476:1123-1132, December 2007. .

Gissinger, C., Ji, H., \& Goodman, J., Instabilities in magnetized spherical Couette ow. to be submitted, 2010.

Goodman J. \& Ji, H., J. Fluid Mech., 462:365, 2002.

Hollerbach, R. \& Rüdiger, G., Phys. Rev. Lett., 95(12):124501-+, 2005. .

Hollerbach, R., Teeluck, V. \& Rüdiger, G., Phys. Rev. Lett., 104(4):044502, 2010.

Ji, H., Magnetorotational instability in a swirling gas and plasma annulus. to be submitted, 2011.

Ji, H., Goodman, J., \& Kageyama, A., Mon. Not. Astron. Soc., 325:L1-L5, 2001.

Ji, H., Burin, M., Schartman, E., \& Goodman, J., Nature., 444:343-346, 2006.

Ji, H., Foley, J., Levinton, F., Fetroe, B., Raitses, Y., Kefeli, J., Nornberg, M., Zweben, S., \& Yamada, M., Bull. Am. Phys. Soc., 52:BAPS.2007.DPP.BP8.86, 2007. 
Kageyama, A., Ji, H., Goodman, J., Chen, F., \& Shoshan, E., J. Phys. Soc. Jpn., 73:2424-2437, 2004.

Lesur G. \& Longaretti, P.-Y., Astron. Astrophys., 444:25-44, 2005.

Lesur G. \& Longaretti, P.-Y., Mon. Not. Astron. Soc., 378:1471-1480, July 2007. .

Liu, W., Phys. Rev. E, 77:056314, 2008.

Liu, W., Goodman, J., Herron, I., \& Ji, H., Phys. Rev. E, 74:056302, 2006.

Liu, W., Goodman, J., \& Ji, H., Phys. Rev. E, 76(1):016310, 2007. .

Lovelace, R., Li, H., Colgate S., \& Nelson, A., The Astrophysical Journal, 513:805, 1999.

Nornberg, M., Ji, H., Schartman, E., Roach, A., \& Goodman, J., Phys. Rev. Lett., 104:074501, 2010.

Paoletti, M. S. \& Lathrop, D. P., ArXiv e-prints 1011.3475, 2010.

Rayleigh, L., Proc. Roy. Soc. London A, 93:148-154, 1916.

Richard, D., PhD thesis, Université Paris 7, 2001.

Richard, D. \& Zahn, Z.-P., Astron. Astrophys., 347:734-738, 1999.

Roach, A., Spence, E., Edlund, E., Sloboda, P. \& Ji, H., Bull. Am. Phys. Soc., 55: BAPS.2010.DPP.NP9.75, 2010.

C. G. Rossby et al. J. Mar. Res, 2(1):38-55, 1939.

Rüdiger, G. \& Hollerbach, R., Phys. Rev. E, 76:068301, 2007.

Rüdiger, G. \& Schultz, M., Astronomische Nachrichten, 331:121, 2010.

Rüdiger, G. \& Zhang, Y., Astron. Astrophys., 378:302-308, 2001.

Schartman, E., Ji, H. \& Burin, M., Rev. Sci, Instrum., 80:024501, 2009.

Schartman, E., Ji, H., Burin, M., \& Goodman, J., Stability of quasi-keplerian shear ow in a laboratory experiment. submitted to Astron. Astrophys., 2010.

Shakura, N. I. \& Sunyaev, R. A., Astron. Astrophys., 24:337-355, 1973.

Sisan, D. R., Mujica, N., Tillotson, W. A., Huang, Y., Dorland, W., Hassam A.B., Antonsen, T. M. \& Lathrop, D. T., Phys. Rev. Lett., 93(11):114502, 2004.

Sommeria, J., Meyers, S. D. \& Swinney, H. L., Nature, 331:689-693, February 1988.

Stefani, F. private communication, 2010.

Stefani, F., Gundrum, T., Gerbeth, G., Rüdiger, G., Schultz, M., Szklarski, J., \& Hollerbach, R., Phys. Rev. Lett., 97:184502, 2006.

Stefani, F., Gerbeth, G., Gundrum, T., Hollerbach, R., Priede, J., Rüdiger, R., \& Szklarski, J., Physical Review E, 80(6):66303, 2009.

Taylor, G. I., Philos. Trans. R. Soc. London, Ser. A, 223:289-343, 1923.

Taylor, G. I., Proc. Roy. Soc. London A, 157:546-578, 1936.

Teodorescu, C., Young, WC., Swan, G. W., Ellis, R. F., Hassam, A. B., \& Romero-Talamas, C. A., Phys. Rev. Lett., 105:085003, 2010.

Velikhov, E. P., Sov. Phys. JETP, 36:995-998, 1959.

Wang, A., Si, J., Liu, W., \& Li, H., Phys. Plasmas, 15:102109, 2008.

Wendt, F., Ing. Arch., 4:577-595, 1933.

Zeldovich, Y. B., Proc. Roy. Soc. London A, 374:299-312, 1981. 